\title{
Management of Aggressive Pituitary Tumors - A 2019 Update
}

Author

Stephan Petersenn

Affiliation

ENDOC, Center for Endocrine Tumors, Hamburg, Germany

Key words

carcinoma, chemotherapy, temozolomide, PRRT,

bevacizumab

received $\quad 03.11 .2019$

accepted $\quad 05.11 .2019$

Bibliography

DOI https://doi.org/10.1055/a-1060-1883

Horm Metab Res 2019; 51: 755-764

(c) Georg Thieme Verlag KG Stuttgart · New York

ISSN 0018-5043

Correspondence

Prof. Dr. Stephan Petersenn

ENDOC Center for Endocrine Tumors

Erik-Blumenfeld-Platz 27a

22587 Hamburg

Germany

Tel.: +49-040-401-87985, Fax:+49-040-401-87986

stephan.petersenn@endoc-med.de

\section{ABSTRACT}

With a prevalence of 80-100/100000, pituitary adenomas are more frequent than thought. The rare aggressive pituitary adenoma presents a special challenge, due to the heterogenous presentation of the disease. The prognosis of aggressive pituitary adenomas has been improved due to recent studies demonstrating some efficacy of chemotherapy with temozolomide. However, there is very limited data on second-line therapies in patients with treatment failure. This review presents an update on the diagnostic and therapeutic management of aggressive pituitary tumors. Patients should be treated by a team consisting of an expert endocrinologist, neurosurgeon, radiation oncologist, and pathologist, and according to the recently published ESE guideline.

\section{Definition of Aggressive Pituitary Tumors}

Pituitary adenomas arise as neoplastic proliferation of cells of the anterior pituitary, with an estimated prevalence of $80-100$ cases per 100000 and an annual incidence of 4 per $100000[1,2]$. The vast majority of pituitary adenomas are of benign nature and do not metastasize. In contrast, the very rare pituitary carcinomas are characterized by the presence of craniospinal and/or systemic metastases, representing $0.12 \%$ of all cases in the German Pituitary Tumor Registry [3]. A minority of pituitary adenomas may develop a clinically aggressive behavior, due to increased cell proliferation and/or invasion in surrounding structures. Of note, invasive behavior alone is not sufficient to define malignancy.

The prevalence of aggressive pituitary adenomas is currently unclear. The 2004 WHO classification of tumors of the pituitary proposed 'atypical adenoma' as a new entity (next to typical adenoma and carcinoma), defined as an invasive tumor with elevated mitotic index, an MiB-1 labeling index $>3 \%$, and an extensive nuclear immunostaining for p53 [4]. This tumor type accounted for $2.7 \%$ of tumors in the German Pituitary Tumor Registry [3]. However, the 2017 WHO classification abandoned this subgroup, as its prognostic significance could not be established [1]. Instead, pituitary adenomas with features that tend to predict recurrence and resistance to conventional therapy are summarized as high-risk pituitary adenomas. Such feature should be rapid growth, radiological invasion, and a high Ki-67 proliferation index. Patients with a combination of these characteristics should be investigated more intensively and followed up more closely.

The 2018 European Society of Endocrinology Clinical Practice Guidelines for the management of aggressive pituitary tumors and carcinomas suggested a more clinically orientated definition of aggressive pituitary tumors [2]. Thereby, the diagnosis should be considered in patients with a radiologically invasive tumor and unusually rapid tumor growth rate, or clinically relevant tumor growth despite optimal standard therapies (surgery, radiotherapy and conventional medical treatments). However, due to the lack of data, neither 'unusually rapid tumor growth rate' nor 'clinically relevant tumor growth' are defined in more detail. Clearly, any definition suggested so far requires clarification and validation by future prospective studies or registries. 


\section{Evaluation}

Baseline and follow-up investigations of all patients with pituitary tumors should be performed in a structured way to recognize aggressive behavior. A careful history is important for early detection of tumor growth and changes in pituitary function. Full endocrine evaluation should evaluate both changes in autonomous hormone secretion in hormonally active pituitary tumors as well as deterioration in pituitary function. Regular MRI scans should define tumor size in all three dimensions [2] with a standardized protocol, with separation of microadenomas $(<1 \mathrm{~cm})$, macroadenomas $(1-4 \mathrm{~cm})$, and giant adenomas $(>4 \mathrm{~cm}$ ) [1]. Furthermore, regular comparison to all previous scans is important to detect minimal but continuous changes during long-term follow-up. Special attention should be given to detect invasion in surrounding structures, as it is a major predictor of aggressive behavior [5]. The Knosp classification offers a standardized and validated approach to describe the invasion of the cavernous sinus space [6]. Frequency of MRI scans should be adapted according to prior tumor growth rate, changes in sign and symptoms, results of endocrine evaluation, and regular evaluation of visual field and neurological deficits, also considering potential side effects of gadolinium administration [7]. Results should be discussed in a multidisciplinary expert team including endocrinologists, radiologists, neurosurgeons, pituitary pathologists, and radiation therapist. Casanueva et al. suggested criteria for the definition of Pituitary Tumor Centers of Excellence which certainly could form the basis for the best interdisciplinary management of aggressive pituitary tumors [8].
Whenever surgical specimens are available, their evaluation according to the current WHO classification is mandatory $[1,9,10]$. The classification requires morphological and immunohistochemical assessment (both for pituitary hormones and transcriptions factors) to define the adenoma type ( $\vee$ Fig. $\mathbf{1})$. Sparsely granulated somatotroph adenoma, lactotroph adenoma in men, Crooke cell adenoma, silent corticotroph adenoma, and plurihormonal PIT1positive adenoma are considered per se as potentially more aggressive tumors and require therefore especially careful follow-up. As Ki-67 was determined as a major predictive factor for aggressive behavior, it should be evaluated in a standardized way [11]. Additional potential markers of aggressiveness as $\mathrm{p} 53$ and markers predictive for medical therapies like expression of somatostatin receptors should be added as needed [1]. As metastases of rare pituitary carcinomas occur in spine, neck lymph nodes, lung, liver and bone, patients with symptoms in those areas or unexplained increases in endocrine markers despite stable pituitary tumor size should undergo site-specific screening [2]. Specimens of metastatic deposits should undergo the same histological evaluation as the primary pituitary tumor.

Trouillas et al. suggested a new clinicopathological classification based on invasiveness, proliferation, and detection of metastases, which was strongly predictive of post-operative remission or tumor progression [5]. It could therefore form the basis to determine follow-up intervals in individual patients.

\begin{tabular}{|c|c|c|c|}
\hline $\begin{array}{l}\text { Adenoma } \\
\text { Types }\end{array}$ & Subtypes & Immunophenotypes & $\begin{array}{l}\text { Transcription } \\
\text { Factors }\end{array}$ \\
\hline \multirow[t]{4}{*}{ Somatotroph } & Densely granulated ${ }^{\star}$ & $\begin{array}{l}\text { GH } \pm P R L \pm a-S U ; C K: \\
\text { diff. }\end{array}$ & Pit-1 \\
\hline & Sparsely granulated & GH \pm PRL; CK: fib bodies & Pit-1 \\
\hline & Mammosomatotroph & GH + PRL (same cell) & Pit-1, ERa \\
\hline & $\begin{array}{l}\text { Somatotroph- } \\
\text { Lactotroph }\end{array}$ & GH+PRL (different cells) & Pit-1, ERa \\
\hline \multirow[t]{3}{*}{ Lactotroph } & Sparsely granulated ${ }^{\star}$ & PRL & Pit-1, ERa \\
\hline & Densely granulated & PRL & Pit-1, ERa \\
\hline & Acidophilic stem cell & PRL, GH (inconstant) & \\
\hline Thyrotroph & Sparsely granulated & $\beta$ TSH, a-subunit & Pit-1, GATA2 \\
\hline \multirow[t]{3}{*}{ Corticotroph } & Densely granulated * & АСTH, СK: +++ & Tpit \\
\hline & Sparsely granulated & АСТН, СК: +++ & Tpit \\
\hline & Crooke's cell & АСТН, СК: +++ & Tpit \\
\hline Gonadotroph & Sparsely granulated ${ }^{\star}$ & $\begin{array}{l}\beta F S H, \beta L H, \alpha-s u b u n i t \\
\text { (various combinations) }\end{array}$ & SF-1, GATA2, ERa \\
\hline Null cell & & None & None \\
\hline \multirow[t]{2}{*}{ Plurihormonal } & Pit-1(Silent subtype 3) & GH, PRL, $\beta$ TSH $\pm a-S U$ & Pit-1 \\
\hline & Unusual ICC combinat. & Various & \\
\hline Double & Distinct adenomas & PRL, ACTH* & Pit-1,Tpit, \\
\hline
\end{tabular}

- Fig. 1 Current classification of pituitary adenomas according to the 2017 WHO report (adapted from [1]) * denotes the most common subtype. 


\section{Therapeutic Options}

\section{Surgery}

Pituitary surgery is the most effective form of treatment for the vast majority of pituitary tumors, except for prolactinomas with their excellent response to dopamine agonists. It intends to normalize pituitary hypersecretory syndromes, eliminate tumor mass and risk of tumor recurrence, while preserving the normal pituitary function and surrounding neural structures. Multiple reports have demonstrated that expert pituitary surgeons with high case numbers have better outcome and less complications than surgeons with fewer pituitary interventions [8]. Therefore, pituitary surgery should always be performed by a dedicated surgeon with extensive experience [2]. MRI grading systems such as the modified Knosp classification may contribute to the prediction of surgical outcome [12]. Patients with clinically aggressive tumor behavior during follow-up should always be re-evaluated by expert neurosurgeons for repeat surgery, especially when primary surgery was performed from a less experienced surgeon. Even if complete removal of the tumor is unlikely, repeated debulking surgery may be helpful to reduce local symptoms like new visual deficiencies or severe headache. Furthermore, endoscopic approaches with enhanced visualization and better access to parasellar structures and into the cavernous sinuses may allow more extensive surgical resection in aggressive pituitary adenomas, thereby avoiding transcranial approaches [13].

\section{Radiotherapy}

Radiotherapy is typically used in patients whose tumor growth and/ or hormonal hypersecretion cannot be controlled with further surgery or medical therapy. Adjuvant radiotherapy may also be considered for residual tumor with pathological markers strongly indicative of aggressive behavior [2]. It should be performed in centers with high experience. Tumor size, location, and previous radiation should be discussed with expert radiation therapists, to determine the most appropriate radiotherapeutic option.

Fractionated external beam radiotherapy (EBRT) relies on different sensitivities of target and surrounding normal tissue to the total accumulated radiation dose [14]. It is delivered in 25-30 daily fractions of 1.8-2.0 Gy over a treatment period of 5-6 weeks, re-

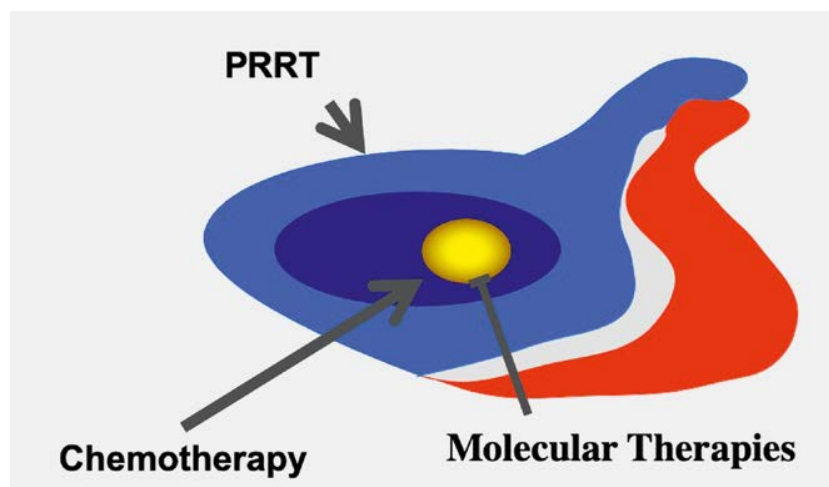

Fig. 2 Therapeutic alternatives in patients failing surgery and radiotherapy sulting in a total radiation dose of 45-54 Gy [15]. In contrast, stereotactic radiosurgery (SRS) aims to eradicate defined target tissue while relatively sparing exposure to adjacent normal tissue due to a steep dose fall-off [14]. Stereotactic guidance by high-resolution imaging allows very precise delivery of radiation to the tumor and is also used with EBRT. SRS is typically applied in a single dose but can be performed in a limited number of sessions, up to a maximum of five (hypo-fractionated radiotherapy). Technologies include linear accelerators (e.g., LINAC and more recently Cyber-knife, a frameless system using robotic mounting and real-time image guidance), multisource Cobalt 60 units (e. g., Gamma Knife), and particle beam accelerators (with limited availability due to the high costs).

There is a clear need for predictive factors identifying those tumors with relevant regrowth potential, to be considered for radiation therapy. Careful radiological evaluation of tumor extension should be performed, as the presence of cavernous sinus extension pre-operatively and suprasellar extension post-operatively are independent predictors of tumor regrowth [16-18]. Moreover, histological and molecular markers may be helpful to identify patients with high risk for tumor recurrence/progression $[11,19,20]$, as has been demonstrated for the proliferation marker Ki-67 in combination with radiological evaluation of invasiveness [5].

Both fractionated EBRT and SRS demonstrate high efficacy rates to control tumor growth, although little data is available concerning their efficacy in more aggressive phenotypes. SRS may be more convenient for the patient with single session therapy compared to daily application of EBRT over several weeks. Some studies claim a more rapid response with respect to biochemical remission and the expectancy of lesser side effects for SRS compared to published data on EBRT. However, to date there are no controlled trials comparing fractionated EBRT and SRS. Due to differences in singles doses some suggestions have been made to choose the appropriate radiation technique. For SRS, the tumor target should be at least 3-5 mm distant from the optic chiasm and less than $3 \mathrm{~cm}$ in diameter. Otherwise, fractionated EBRT may be the only option. Furthermore, EBRT should be preferred for tumors with irregular anatomy, including diffuse local infiltration and suprasellar or brainstem extension, to avoid high dose radiation of healthy tissue [15]. Of note, SRS has been used as salvage therapy with some success in a small series of patients with persistent active tumors despite prior fractionated EBRT [21].

The decision for radiotherapy must be balanced against potential side effects. Early side effects include nausea and lassitude (usually mild, lasting $<2$ months), diminished taste and olfaction ( $<6$ months), and hair loss at entry sites (< 1 year) [22], with long-term side effects include hypopituitarism, cerebrovascular disease, secondary tumor formation, damage to surrounding structures like the optic chiasm, and neurocognitive dysfunction.

\section{Chemotherapy}

\section{Temozolomide}

In patients failing surgery and radiation with ongoing tumor progression or detection of metastases, systemic therapy may be required ( $\triangleright$ Fig. 2). After initial case reports on the use of temozolomide in single patients with aggressive pituitary tumors in 2006 
[23-25], subsequent series have confirmed the efficacy of temozolomide in patients with aggressive pituitary tumors.

In a careful meta-analysis on 57 patients, presented in case reports $(55$ patients from 29 publications plus 2 additional patients by the authors, with updated follow-up on 22 patients from 9 publications) the objective response rates for aggressive pituitary adenomas and pituitary carcinomas were $48.4 \%$ and $65.2 \%$, respectively [26]. Disease stabilization occurred in 29.0 and $17.4 \%$, respectively. The median duration of response was 30 months (5.5-120). Patients with long-term treatment $>12$ months (35.7\%) demonstrated longer PFS than those on short-term treatment, although the difference was statistically not significant.

Subsequent to single case reports, a number of larger series has been published during the last 10 years. As small studies carry a higher risk of publication bias, only studies including more than 3 patients will be discussed below in more detail.

In a prospective 1-year treatment study on 6 consecutive patients with aggressive pituitary adenomas by Losa et al., 4 patients completed 12 cycles of temozolomide $\left(150-200 \mathrm{mg} / \mathrm{m}^{2}, 5 / 28 \mathrm{~d}\right.$ ) with 1 complete and 1 partial remission, and 2 patients with stable disease (follow-up of 28, 12, 24, and 21 months, respectively). Two patients demonstrated progress after 3 and 6 months [27]. In a retrospective evaluation of 7 patients treated with temozolomide $\left(75 \mathrm{mg} / \mathrm{m}^{2}, 21 / 28 \mathrm{~d}\right)$ reported by Bush et al., 2 patients responded with partial remission ( 1 ongoing after 11 cycles with further treatment, 1 ongoing after 11 cycles with subsequent surgery due to CSF leakage and possibly related to gamma knife therapy 5 months prior to temozolomide), 4 patients with disease stabilization ( 3 patients with ongoing treatment after 10,10 , and 13 cycles, 1 patient for 2 cycles), and 1 patient with progressive disease [28]. In a retrospective multicenter evaluation from France, Raverot et al. provided details on 8 patients treated with temozolomide $\left(150-200 \mathrm{mg} / \mathrm{m}^{2}, 5 / 28 \mathrm{~d}\right)$ for $3-24$ cycles [29]. Partial remission was described in one lactotroph carcinoma and two corticotroph tumors (one aggressive adenoma, one carcinoma). In a retrospective multicenter evaluation from Japan, Hirohata et al. described 13 patients treated with temozolomide (mostly $150-200 \mathrm{mg} / \mathrm{m}^{2}$, $5 / 28$ d) [30]. Complete remission was seen in 3 patients ( 1 with a recurrence after 10 months during ongoing treatment), partial remission in 6 patients (with recurrences in 5 patients after 5-19 months during ongoing treatment), stable disease in 2 patients, and progressive disease in 2 patients. Bengttson et al. published retrospective data on 24 patients treated with temozolomide (150$200 \mathrm{mg} / \mathrm{m}^{2}, 5 / 28 \mathrm{~d}$ ) for a median of 6 months (1-23) [31]. Conclusive follow-up data was available for 21 patients, with 2 complete remissions (ongoing at 48 and 91 months), 8 partial remissions ( 2 ongoing at 31 and 81 months (off therapy), 1 progress at 21 months (off therapy), 1 progress at 6 months (during therapy), 2 with 2 nd course of therapy at 4 and 20 months), stable disease in 3 patients [ongoing at 17, 30, and 44 months (off therapy)], and 8 patients with progressive disease. Ceccato et al. reported their retrospective single-center experience with 5 patients treated with temozolomide (150-200 mg/m², 5/28 d) for a median of 12 months (324), with partial remission in 2 patients (for 12 and 24 months), stable disease in 1 patients for $>6$ months, and progressive disease in 2 patients [32]. Bruno et al. described 6 patients studied retrospectively after treatment with temozolomide $(140-320 \mathrm{mg}, 5 / 28 \mathrm{~d})$, with objective response in 2 patients [ongoing at 31 months and 59 months (off therapy)] [33]. Losa et al. presented the results of a retrospective multicenter survey in Italy, with 31 patients treated with temozolomide $\left(150-200 \mathrm{mg} / \mathrm{m}^{2}, 5 / 28 \mathrm{~d}\right.$, except for 2 patients following the Stupp protocol) [34]. Partial remission was seen in 11 patients, stable disease in 14 patients, and progressive disease in 6 patients. During follow-up of 43 months (24-72), 13 patients of those with at least prior disease stabilization demonstrated regrowth of the tumor. Lasolle et al. presented the results of a second retrospective survey from France on 43 patients [35], including follow-up on some patients presented in the first survey. Most patients were treated with temozolomide $150-200 \mathrm{mg} / \mathrm{m}^{2}(5 / 28 \mathrm{~d})$, whereas 6 patients were treated according to the Stupp protocol. At least partial remission according to tumor and/or hormonal changes was found in 22 patients (with relapse during follow-up in 10 patients), stable disease in 10 patients (with progress during follow-up in 4), and progressive disease in 11 patients. In a retrospective single-center evaluation from Boston, Jordan et al. described the outcome for 7 patients [36]. Most patients received temozolomide $150-200 \mathrm{mg} / \mathrm{m}^{2}(5 / 28 \mathrm{~d})$, with 2 patients increasing their dose with tumor progression either up to $200 \mathrm{mg} / \mathrm{m}^{2}$ daily on alternate weeks or $75 \mathrm{mg} / \mathrm{m}^{2}$ daily. Tumor regression was seen in 4 patients, and disease stabilization in 3 patients. Median PFS was 1.6 years.

Most recently, McCormack et al. published the results of a retrospective European Society of Endocrinology (ESE) survey on 157 patients treated with temozolomide for a median of 9 months (1-36) [37]. The vast majority of patients ( $93 \%$ ) were treated according to the standard protocol with $150-200 \mathrm{mg} / \mathrm{m}^{2}$ (5/28 d), $4 \%$ received treatment according to the Stupp protocol, with the remaining few patients treated according to a variety of protocols, including some dose-dense regimes. According to tumor response, 9 (6\%) patients demonstrated complete regression, 49 (31\%) partial regression, 52 (33\%) stable disease, and 47 (30\%) progressive disease. The overall radiological response rate was $37 \%$. Regression was observed more frequently in clinically functioning compared to nonfunctioning tumors $(p=0.01)$. Response to temozolomide was significantly higher in patients with concomitant radiotherapy $(p=0.02)$. During a median follow-up of 21 months $(0-102)$ after drug cessation, $25 \%, 37 \%$, and $41 \%$ patients with prior complete remission, partial remission, or stable disease, respectively, developed a relapse [median time to progress 12 (1-60) months after drug cessation] ( $\vee$ Fig. $\mathbf{3}$ ).

Altogether, those 11 studies comprise 304 patients, with objective response in 125 patients (41.1\%), and disease stabilization in additional 91 patients (29.9\%). However, it has to be stressed, that a relevant number of patients experienced relapses and therefore require additional therapies. Furthermore, it remains unclear, whether continuous long-term treatment offers any advantage compared to short-term treatment.

\section{Other forms of chemotherapy}

Due to the rarity of pituitary carcinomas few studies have been published on the use of intravenous chemotherapy [38]. The recent European Society of Endocrinology (ESE) survey lists a variety of substances (cisplatin, carboplatin, oxaliplatin, etoposide, adriblastin, capecitabine, 5FU, doxorubicine, cyclophosphamide) applied 


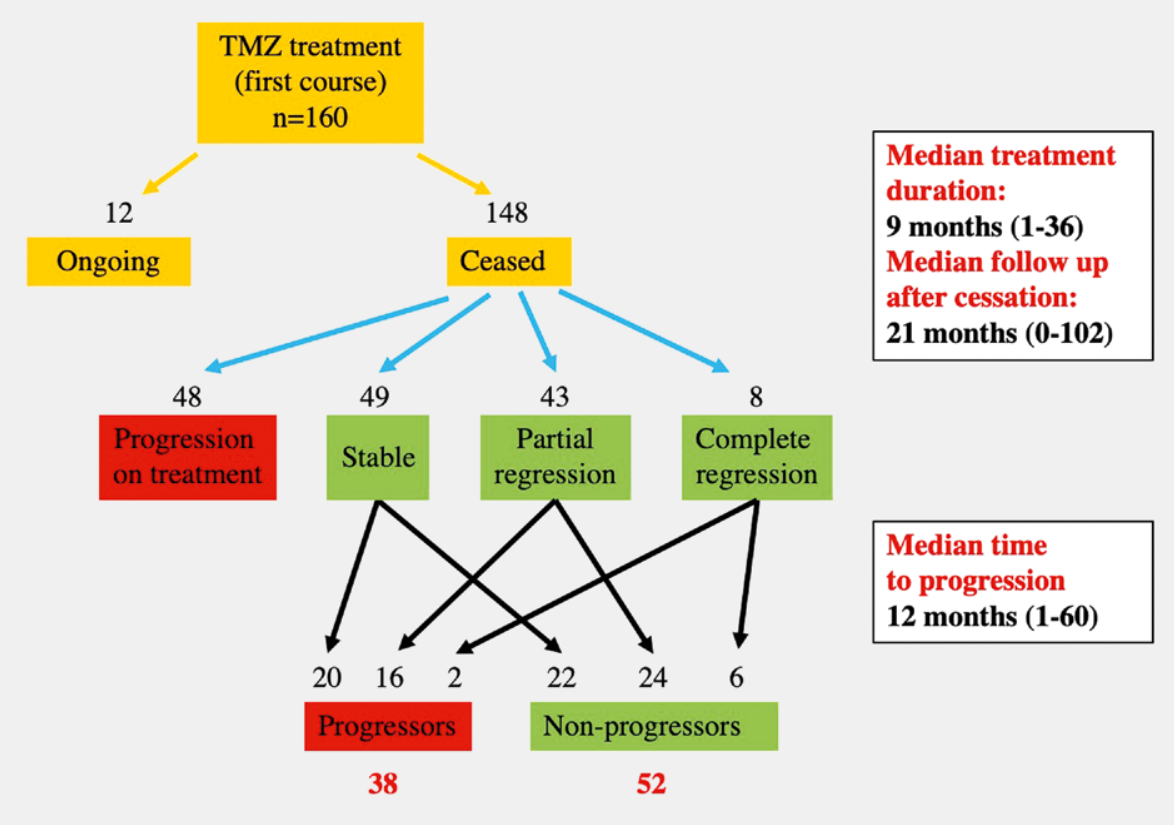

- Fig. 3 Results of a recent European Society of Endocrinology (ESE) survey on the treatment of aggressive pituitary adenomas and carcinomas: efficacy of temozolomide and rate of recurrence during follow-up after cessation of temozolomide (number of patients shown) (adapted from [37]).

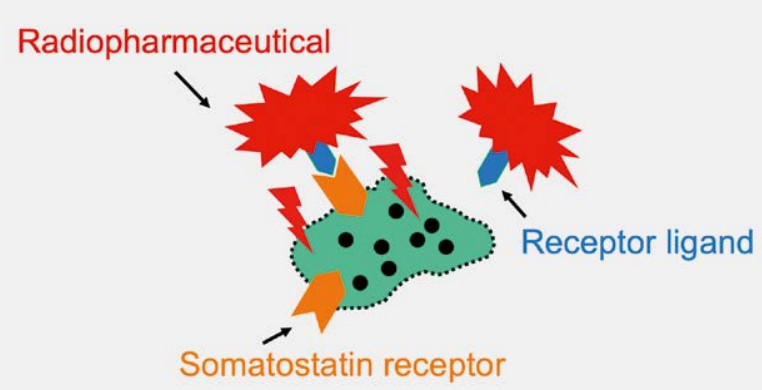

- Fig. 4 Principles of peptide receptor radionuclide therapy (PRRT): Radiopharmaceuticals are linked to the receptor ligand, which binds to its specific receptor at the surface of the tumor cell. Radioactivity is thereby specifically transported to its target cells.

as second and third line therapies and in various combinations in very few patients [37]. In one of the few studies summarizing experience with one scheme, Kaltsas et al. presented their institutional experience with a combination of lomustine $100 \mathrm{mg} / \mathrm{m}^{2}$ orally on $\mathrm{d} 1$ and $5 \mathrm{FU}$ (each $400 \mathrm{mg} / \mathrm{m}^{2}$ as iv bolus followed by iv infusion over 22 h) on $d 1+d 2$ every 3 weeks [39]. Seven patients with aggressive pituitary adenomas or pituitary carcinomas were treated for a median of 2 cycles (range 1-6), with clinical improvements in 3 patients, biochemical improvements in 2 patients, and objective tumor size reduction in 1 patient. Toxicity was minimal.

\section{Experimental therapies \\ PRRT}

Peptide receptor radionuclide therapy (PRRT) has been successfully used for the treatment of various neuroendocrine tumors $[40,41]$, with acceptable side effects. It is usually based on the high expression of somatostatin receptors in endocrine tumor cells. Radiopharmaceuticals linked to the receptor ligand are able to deliver focused therapy to the tumor ( $\$$ Fig. 4 ). In vivo, expression of somatostatin receptors can be evaluated by ${ }^{111}$ In-octreotide-scintigraphy, to potentially predict the applicability of PRRT in individual patients. A PubMed search revealed 12 studies with relevant patient numbers (excluding case reports) as well as sufficient data on pituitary adenoma types and uptake in 367 patients ( $\bullet$ Table 1 ) [42-53]. More than two third of patients with $\mathrm{GH}$ - or TSH-expressing pituitary adenomas, half of the patients with non-functioning or ACTH-secreting adenomas, and approximately $40 \%$ of patients with PRL-expressing adenomas demonstrated positive uptake of ${ }^{111}$ In-octreotide. Interestingly, Acosta-Gomez et al. confirmed relevant uptake also in a subgroup of patients with recurrent adenomas [42].

68Ga-DOTA-TOC and -TATE PET represent major advances in comparison to ${ }^{111}$ In-octreotide-scintigraphy, offering better resolution and quantitative assessment of somatostatin receptor expression, and may be combined with either CT or MRI scan. Tissue uptake for 68Ga-DOTA-TOC exclusively correlated with quantitative expression of sst2 [54]. Several case reports have demonstrated strong tracer uptake in pituitary tumors, for example, as rare combination with multiple paragangliomas [55], to identify tumor boundaries of a residual adenoma more precisely than by MRI alone prior to cyberknife radiotherapy [56], to screen for metastatic disease and thereby confirm the diagnosis of a pituitary carcinoma 
- Table 1 Details on expression of somatostatin receptors investigated by ${ }^{111} \mathrm{In}$-octreotide-scintigraphy in various pituitary adenomas.

\begin{tabular}{|c|c|c|c|c|c|}
\hline Reference & $\mathrm{NFA}+\mathrm{LH}+\mathrm{FSH}$ & PRL & Acro & Cush & TSH \\
\hline Acosta-Gomez BJR 2005 [42] & $2 / 5$ & $3 / 4$ & $4 / 4$ & $0 / 3$ & $1 / 1$ \\
\hline Acosta-Gomez BJR 2005 (recurrence) [42] & $5 / 7$ & $3 / 7$ & $1 / 3$ & $4 / 5$ & - \\
\hline Boni Q J Nucl Med 1995 [43] & $2 / 12$ & - & $12 / 13$ & - & $2 / 4$ \\
\hline Borson-Chazot Clin Endocrinol 1997 [44] & $16 / 29$ & - & $12 / 19$ & - & - \\
\hline Colao J Endocrinol Invest 1999 [45] & $9 / 9$ & - & $21 / 29$ & - & - \\
\hline Duet J Nucl Med 1999 [46] & $13 / 17$ & & $9 / 14$ & & $6 / 6$ \\
\hline Goerges Nuklearmedizin 1997 [47] & - & - & $14 / 22$ & - & - \\
\hline Legovini J Endocrinol Invest 1997 [48] & - & - & $8 / 9$ & - & - \\
\hline Oppizzi J Endocrinol Invest 1998 [49] & $14 / 22$ & & $14 / 17$ & & \\
\hline Ploeckinger JCEM 1994 [50] & $4 / 12$ & - & $4 / 7$ & - & - \\
\hline Rieger Neurosurg Rev 1997 [51] & $9 / 25$ & $1 / 6$ & $5 / 11$ & - & - \\
\hline Schmidt Eur J Nucl Med [52] & $1 / 15$ & $1 / 2$ & $1 / 8$ & - & - \\
\hline Tofani Q J Nucl Med 1995 [53] & $5 / 7$ & $1 / 5$ & $7 / 8$ & - & - \\
\hline Total & $80 / 160$ & $9 / 24$ & $112 / 164$ & $4 / 8$ & $9 / 11$ \\
\hline Total (\%) & 50.0 & 37.5 & 68.3 & 50.0 & 81.8 \\
\hline
\end{tabular}

[57] or to determine a pituitary carcinoma as the primary lesion in a patient with lung metastasis of a neuroendocrine tumor [58], to clarify the diagnosis of a giant prolactinoma in combination with a meningioma [59], to confirm a recurrence of a small TSH-secreting pituitary adenoma in an empty sella as depicted by MRI scan [60], or to localize an ectopic TSH-secreting pituitary adenoma in the nasopharynx [61].

Zhao et al. evaluated the combined use of ${ }^{68} \mathrm{Ga}$-DOTA-TATE PET/ CT and FDG PET/CT in 35 patients with residual or recurrent pituitary adenomas, both performed within one week prior to surgery [62]. Tumor tissue in 34/35 adenomas demonstrated relevant, but variable uptake by ${ }^{68} \mathrm{Ga}$-DOTA-TATE PET, with generally higher uptake in the remaining normal pituitary tissue. Similar results were obtained in 37 patients with suspicion of pituitary microadenomas investigated by ${ }^{68} \mathrm{Ga}-\mathrm{DOTA}-\mathrm{TATE}$ PET/MRI and FDG PET/MRI [63]. Pituitary insufficiency may therefore be a concern in patients treated by PRRT.

Despite multiple evidence of somatostatin receptor expression in vitro and in vivo, PRRT has rarely been applied for the treatment of pituitary tumors, except for a few case reports. Baldari et al. demonstrated the effectiveness of PRRT with ${ }^{111}$ In-DTPA-octreotide in a giant PRL-secreting pituitary adenoma resistant to conventional treatment [64]. A 58 year-old woman with severe neurological symptoms, increasing prolactin levels and tumor size despite prior surgery, medical treatment with cabergolin and radiotherapy was investigated by ${ }^{111}$ In-octreotide-scintigraphy, revealing strong uptake of the tracer. Subsequent medical treatment with octreotide LAR was ineffective. The patient underwent four cycles of PRRT with ${ }^{111}$ In-DTPA-octreotide (cumulative activity $29 \mathrm{GBq}$ ), with substantial tumor shrinkage and significant improvement in clinical conditions. According to the report she was still in stable condition 2 years after the beginning of the PRRT; no side effects were reported. Kumar Gupta et al. reported a 71-year old woman with a pancreatic neuroendocrine tumor [65]. Further investigation by ${ }^{68} \mathrm{Ga}-$ DOTA-NOC PET/CT excluded distant metastases but revealed intense intracranial radiotracer uptake corresponding to a nonfunctioning pituitary macroadenoma and suggesting MEN-1. The patient opted for experimental PRRT with ${ }^{177}$ Lu-DOTATATE (150 $\mathrm{mCi}$ ), with subsequent decrease of chromogranin A. However, no data were given on follow-up of the pituitary adenoma. Kovacs et al. described a 16-year old female with an aggressive ACTH-secreting macroadenoma [66]. The patient underwent eight pituitary surgeries, bilateral adrenalectomy, and three courses of radiation therapy. As ${ }^{111}$ In-octreotide-scintigraphy revealed positive uptake of a progressively growing tumor remnant, the patient received two cycles of ${ }^{90}$ Yttrium-DOTATATE (200 mCi). Imaging showed increased radionuclide uptake at the left side of the neck, and whole-body CT found additional metastases in the liver. The patient died within the following year of elevated intracranial pressure. Komor et al. published a 55-year-old patient with right-sided headache and subsequent diagnosis of a nonfunctioning pituitary adenoma with infiltration into the right cavernous sinus [67]. Histology revealed a null-cell adenoma with high $\mathrm{Ki}-67$ of $12 \%$, and homogenous expression of sst2 by somatostatin receptor autoradiography. Following radiosurgery the patient remained stable for 8 years, after which he presented with an incomplete palsy of the right oculomotorius nerve due to relevant increase of the tumor remnant. With positive ${ }^{111}$ In-octreotide-scintigraphy, the patient underwent 3 cycles of ${ }^{177} \mathrm{Lu}$-DOTATOC (each $200 \mathrm{mCi}$ ). The palsy of the oculomotorius nerve improved and the patient remained stable for more than 8 years at the time of the report. MacLean reported on 3 consecutive patients referred for ${ }^{68} \mathrm{Ga}$-DOTATATE PET/CT to evaluate 
suitability for ${ }^{177}$ Lu-DOTATATE therapy [68]. A 67-year old male underwent surgery and adjuvant radiotherapy for a nonfunctioning pituitary adenoma, with second surgery for a recurrence 19 years later. Another 3 years later, he was diagnosed with metastatic disease of his spine and skull, and underwent 4 cycles of ${ }^{177} \mathrm{Lu}-\mathrm{DO}$ TATATE ( 7.4 GBq each). Except for a temporary drop in his platelet count he experienced no severe side effects and his disease remained stable and symptom-free 40 months after treatment induction. A second case, a 42 year-old man presented with diplopia due to an invasive $\mathrm{GH}$ - and $\mathrm{Prl}$-secreting pituitary adenoma. $\mathrm{He}$ was treated by surgery followed by radiotherapy and medical therapy with lanreotide and cabergoline. In the following 3 years he underwent 4 additional surgeries, 2 cycles with temozolomide, and radiosurgery. With continuous progress causing bilateral ophthalmoplegia, chiasm compression, and ptosis as well as brainstem compression, he was investigated by ${ }^{68} \mathrm{Ga}$-DOTATATE PET/CT, demonstrating strong uptake. He received 2 cycles of ${ }^{177}$ Lu-DOTATATE (7.8 and 7.5 GBq) but died shortly afterwards due to deterioration of his brainstem disease. A third case, a 32-year old, had transsphenoidal surgery for a silent corticotroph adenoma (retrospective with increased proliferation markers), with second surgery and radiotherapy for recurrent disease 2 years later, another debulking surgery one year later, followed by 6 cycles of temozolomide. He received one cycle of ${ }^{177}$ Lu-DOTATATE, but with severe facial pain was then treated by chemotherapy, additional surgeries and radiotherapy during the following year. Despite radiological response to the later, he then died suddenly. Novruzov et al. described a 68-year old male with diagnosis of a nonfunctioning pituitary carcinoma and spinal metastases 20 years after surgery and radiation therapy for a pituitary macroadenoma [69]. The patient received 3 cycles of ${ }^{177} \mathrm{Lu}$-DOTATATE ( $7.4 \mathrm{GBq}$ each cycle), and subsequently remained stable during the follow-up of 4 years. Waligórska-Stachura et al. presented a 26-year male with a giant $\mathrm{GH}$-secreting pituitary adenoma, with uncontrolled disease despite prior transsphenoidal and transcranial surgery, medical treatment with SLR, and radiation therapy [70]. After confirming somatostatin receptor expression by ${ }^{68} \mathrm{Ga}$-DOTATATE PET/CT, he was treated with 4 cycles of ${ }^{90}$ Yttrium-DOTATATE ( $100 \mathrm{mCi}$ every 3 months), with subsequent tumor regression and biochemical control during follow-up of 12 months. Most recently, Giuffrida et al. presented 3 cases treated by PRRT [71]. A 55-year old female presented with a rapidly growing pituitary mass and increasing prolactin levels, after diagnosis of a macroprolactinoma 13 years earlier resistant to treatment with cabergolin but controlled by transsphenoidal surgery. Radiotherapy of the aggressive prolactinoma was stopped due to rapid worsening of the clinical condition, including neurological impairment and left oculomotor nerve palsy. She received five cycles of PRRT with ${ }^{111}$ In-DTPA-octreotide (cumulative activity $37 \mathrm{GBq}$ ), with remarkable tumor shrinkage and decrease of her prolactin levels, as well as relevant improvement in clinical condition. She remained stable during follow-up over 96 months, without relevant side effects. The second case, a male with a giant prolactinoma, demonstrated ongoing progress of his tumor despite three surgeries and hypo-fractionated radiosurgery. He received 2 cycles of ${ }^{177}$ Lu-DOTATOC (12.6 GBq), with a dramatic increase in tumor size shortly after the 2 nd cycle, and subsequently underwent chemotherapy with temozolomide and cyclophospha- mide, without any benefit but progressive neurological symptoms. The third case, a female with a giant nonfunctioning pituitary adenoma treated by 5 surgeries, fractionated radiotherapy and chemotherapy with temozolomide over 10 years, received 5 cycles of ${ }^{177}$ Lu-DOTATOC (29.8 GBq), but demonstrated clear progress of her tumor mass and deterioration of clinical symptoms over the following year. Two recent reports on the efficacy of temozolomide mentioned 5 patients receiving PRRT $[31,35]$, but as details were scarce and overlap with the case reports could not be excluded, data are not presented here.

Altogether, these case reports combine data on 12 patients ( 9 adenomas, 3 carcinomas; 3 PRL, 5 NFA, 1 GH/PRL, 1 GH, 2 ACTH), of which 6 responded to PRRT with disease stabilization or partial remission of the tumor during a median follow-up of 44 months (range 1-8 years). Five patients were considered non-responders, and 1 patient lacked sufficient follow-up data. Most patients were treated by ${ }^{177}$ Lu-DOTATOC or ${ }^{177}$ Lu-DOTATATE $(n=8)$, whereas 2 patients received ${ }^{111} \mathrm{In}$-DTPA-octreotide and 2 patients ${ }^{90}$ YttriumDOTATATE. Although selection of those case reports may be biased, data appears to be sufficient to evaluate patients without therapeutic alternatives for expression of somatostatin receptors.

\section{Molecular therapies}

Increasing knowledge on cell signaling and molecules involved in cell proliferation has evolved in the successful development of new cancer therapies. Subsequently, several studies have investigated their potential for the treatment of aggressive pituitary adenomas and pituitary carcinomas, mostly at the experimental level. Few patients resistant to other therapies have been treated with those compounds, and published data on their follow-up will be summarized below (selection limited to cases with sufficient data on treatment and follow-up).

\section{Potential of anti-VEGF therapy}

Bevacizumab is a humanized monoclonal antibody directed against vascular endothelial growth factor (VEGF). Is has been approved as an anti-angiogenic treatment for various forms of cancers mostly in combination with chemotherapy. Ortiz et al. described a case of a silent corticotroph cell carcinoma, with repeated sellar tumor regrowth despite 7 surgeries, radiotherapy, and 3 courses of temozolomide, and accompanied by the development of 2 vertebral metastases treated by surgery and focal radiotherapy [72]. As the tumor demonstrated conclusive VEGF immunoreactivity, the patient received intravenous bevacizumab $10 \mathrm{mg} / \mathrm{kg}$ every 2 weeks for the follow-up of 26 months, with ongoing disease stabilization. Touma et al. reported a case with a corticotroph carcinoma and pulmonary metastasis, treated by pituitary surgery followed by combined radiation, temozolomide $75 \mathrm{mg} / \mathrm{m}^{2}$ daily and bevacizumab $10 \mathrm{mg} / \mathrm{kg}$ every 2 weeks for 8 weeks [73]. At that time, the pulmonary nodule had dissolved, TMZ was continued with $200 \mathrm{mg} /$ $\mathrm{m}^{2}$ (5/28 d) for a total of 12 cycles, and the patient remained in remission during the follow-up of 5 years. Rotman et al. published a case of a corticotroph carcinoma with distant metastases in the CNS, after initial diagnosis of a corticotroph adenoma 14 years earlier treated by surgery and radiation [74]. The patient underwent surgery for a temporal cystic mass, hypofractionated radiotherapy of a cervicomedullary metastasis, followed by 12 cycles of adjuvant 
temozolomide (150-200 mg/m² (5/28 d) overlapping with bevacizumab $10-15 \mathrm{mg} / \mathrm{kg}$ every 2 weeks for 2 years. He thereby remained progression-free during the follow-up of 8 years. Dutta et al. presented a 4-year old boy with a giant somatotroph adenoma due to a germline AIP mutation, with immediate progress of a large tumor remnant after surgery [75]. The patient was immediately treated by temozolomide $180 \mathrm{mg} / \mathrm{m}^{2}$ (5/28 d for 38 months), followed 3 months later by fractionated radiotherapy and initiation of bevacizumab $5-10 \mathrm{mg} / \mathrm{kg}$ every 2 weeks (for 35 months), with clear tumor shrinkage but continuous $\mathrm{GH}$ excess (intermittently treated by octreotide LAR and/or pegvisomant). He then underwent gamma knife radiotherapy with parallel treatment with octreotide LAR and pegvisomant.

\section{Treatment by mTOR inhibition}

Activation of the mTOR pathway is common in human neoplasia and has also been described in pituitary tumors. The mTOR inhibitor everolimus is approved for a variety of cancer and has demonstrated clear efficacy in pancreatic neuroendocrine tumors. Jouanneau et al. described a case of an initially silent corticotroph carcinoma with 2 pituitary surgeries, radiotherapies of the sellar region and subarachnoid metastasis, bilateral adrenalectomy and temozolomide $200 \mathrm{mg} / \mathrm{m}^{2}$ (5/28 d) [76]. With continuous progression, the patient received salvage therapy with everolimus orally $5 \mathrm{mg} / \mathrm{d}$ for 3 months combined with octreotide LAR $30 \mathrm{mg}$ i.m. every 28 days (stopped after 1 month due to side effects), without any effect and death of the patient 5 months later. Donovan et al. presented a case of a corticotroph carcinoma with progressive disease despite 6 surgeries, radiation therapy, bilateral adrenalectomy, and chemotherapy with capecitabine and temozolomide [77]. As next generation sequencing revealed a STK11 mutation in the MTOR pathway, she was started on everolimus $7.5-10 \mathrm{mg} / \mathrm{d}$, with parallel palliative radiation of bone metastases. She stabilized for $>6$ months, but eventually developed systemic progression and died shortly afterwards. Zhang et al. published a case with an aggressive lactotroph adenoma with progressive tumor growth despite 2 surgeries, radiation therapy and treatment with cabergoline in increasing doses [78]. As he declined chemotherapy with temozolimide, he was started on everolimus $10 \mathrm{mg} / \mathrm{d}$ combined with cabergoline $1.5 \mathrm{mg} / \mathrm{d}$, leading to disease stabilization for 12 months, with subsequent rise in prolactin levels.

\section{Effects of tyrosine kinase inhibitors}

Activation of tyrosine kinase receptors and their pathways has been implicated in the pathogenesis of endocrine tumors. Subsequently, tyrosine kinase inhibitors have been approved for the treatment of a variety of endocrine tumors, for example, neuroendocrine tumors and medullary thyroid carcinomas. Given the relevance of EGF receptor signaling for the control of lactotroph cells, Cooper et al. treated 2 patients with lactotroph adenomas resistant to dopamine agonist therapy with lapatinib, a tyrosine kinase inhibitor with effects on EGF receptor signaling $(1250 \mathrm{mg}$ orally for 6 months) [79]. Subject 1 with a giant tumor treated by surgery and dopamine agonists but increasing tumor size responded to lapatinib with a $78 \%$ and $22 \%$ decrease in prolactin levels and tumor size, respectively, with symptomatic improvements and mild side effects. Therapy was continued as part of a compassionate use pro- gram. Subject 2 with a macroprolactinoma treated by surgery and dopamine agonists but persistent tumor growth responded to lapatinib with a $42 \%$ decrease in prolactin, stabilization of tumor size and clinical improvements.

\section{Use of checkpoint inhibitors}

CTLA-4 and PD-1 are important inhibitors of the immune system. As cancer cells utilize those proteins to evade anti-tumor responses, inhibitor of CTLA-4 (ipilumab) and PD-1 (nivolumab) have been developed (so called checkpoint inhibitors), which clearly improved survival for a variety of cancers. Lin et a. presented a case of a corticotroph carcinoma with continuous progress after 4 surgeries, 2 radiotherapies, bilateral adrenalectomy, 2 courses of chemotherapy with capecitabine and temozolomide, and chemotherapy with carboplatin and etoposide [80]. At that time she received investigational treatment with ipilimumab ( $3 \mathrm{mg} / \mathrm{kg}$ every 3 weeks) and nivolumab (1 mg/kg every 3 weeks), with immediate 10 -fold decrease of ACTH within 1 week, followed by $92 \%$ and $59 \%$ reductions in size of her hepatic metastasis and recurrent intracranial component, respectively. She received 5 cycles of combined treatment and was then switched to maintenance therapy with nivolumab, remaining stable during the follow-up of 6 months.

\section{Summary}

The recent European Society of Endocrinology Clinical Practice Guidelines for the management of aggressive pituitary tumors and carcinomas [2] provide an important and clinically relevant basis for the management of patients with this rare but difficult to treat disease. In contrast to the usual benign behavior of pituitary adenomas, a subset develops an aggressive course, sometimes even transforming into carcinomas. Until recently, the therapeutic options were very limited, after surgery and radiotherapy failed. Fortunately, data is accumulating on the use of temozolomide as oral chemotherapy with relatively good tolerability. However, important questions remain unsolved: How long should temozolomide treatment be continued? Preferable combined with radiotherapy? Are there any reliable markers to predict treatment efficacy (with evaluation of mgMT status)? And what are second line options in those patients failing temozolomide?

A number of new therapies have emerged, improving the survival in various form of cancers. Unfortunately, data on their use in aggressive pituitary adenomas and carcinomas are limited to single case reports. Future studies should be carefully designed as multicenter trials or part of large registries, to include patients in a prospective way and generate meaningful data, so that the still very limited prognosis may be improved.

\section{Conflict of Interest}

The author declares that he has no conflict of interest. 
[1] Lloyd RV, Osamura RY, Klöppel G et al. World Health Organization Classification of Tumours of Endocrine Organs. World Health Organization. 4th edition. Lyons: IARC; 2017

[2] Raverot G, Burman P, McCormack A et al. European Society of Endocrinology Clinical Practice Guidelines for the management of aggressive pituitary tumours and carcinomas. Eur J Endocrinol 2018; 178: G1-G24

[3] Saeger W, Ludecke DK, Buchfelder M et al. Pathohistological classification of pituitary tumors: 10 years of experience with the German Pituitary Tumor Registry. Eur J Endocrinol 2007; 156: 203-216

[4] DeLellis RA, Lloyd RV, Heitz PU et al. World Health Organization Classification of Tumours of Endocrine Organs. World Health Organization. 3rd edition. Lyons: IARC; 2004

[5] Trouillas ], Roy P, Sturm $\mathrm{N}$ et al. A new prognostic clinicopathological classification of pituitary adenomas: A multicentric case-control study of 410 patients with 8 years post-operative follow-up. Acta Neuropathol 2013; 126: 123-135

[6] Knosp E, Steiner E, Kitz K et al. Pituitary adenomas with invasion of the cavernous sinus space: A magnetic resonance imaging classification compared with surgical findings. Neurosurgery 1993; 33: 610-617. Discussion 617

[7] Nachtigall LB, Karavitaki N, Kiseljak-Vassiliades K et al. Physicians' awareness of gadolinium retention and MRI timing practices in the longitudinal management of pituitary tumors: A "Pituitary Society" survey. Pituitary 2019; 22: 37-45

[8] Casanueva FF, Barkan AL, Buchfelder M et al. Criteria for the definition of Pituitary Tumor Centers of Excellence (PTCOE): A Pituitary Society Statement. Pituitary 2017; 20: 489-498

[9] Saeger W, Honegger ], Theodoropoulou M et al. Clinical impact of the current WHO classification of pituitary adenomas. Endocr Pathol 2016; 27: 104-114

[10] Saeger W, Petersenn S, Schofl C et al. Emerging histopathological and genetic parameters of pituitary adenomas: Clinical impact and recommendation for future WHO classification. Endocr Pathol 2016; 27: $115-122$

[11] Miermeister CP, Petersenn S, Buchfelder M et al. Histological criteria for atypical pituitary adenomas - data from the German pituitary adenoma registry suggests modifications. Acta Neuropathol Commun 2015; 3: 50

[12] Buchy M, Lapras V, Rabilloud M et al. Predicting early post-operative remission in pituitary adenomas: evaluation of the modified knosp classification. Pituitary 2019; 22: 467-475

[13] Heaney A. Management of aggressive pituitary adenomas and pituitary carcinomas. J Neurooncol 2014; 117: 459-468

[14] Barnett GH, Linskey ME, Adler JR et al. Stereotactic radiosurgery - an organized neurosurgery-sanctioned definition. J Neurosurg 2007; 106: 1-5

[15] Ding D, Starke RM, Sheehan JP. Treatment paradigms for pituitary adenomas: Defining the roles of radiosurgery and radiation therapy. J Neurooncol 2014; 117: 445-457

[16] Brochier S, Galland F, Kujas M et al. Factors predicting relapse of nonfunctioning pituitary macroadenomas after neurosurgery: A study of 142 patients. Eur J Endocrinol 2010; 163: 193-200

[17] Chang EF, Zada G, Kim S et al. Long-term recurrence and mortality after surgery and adjuvant radiotherapy for nonfunctional pituitary adenomas. J Neurosurg 2008; 108: 736-745

[18] Greenman Y, Ouaknine G, Veshchev I et al. Postoperative surveillance of clinically nonfunctioning pituitary macroadenomas: Markers of tumour quiescence and regrowth. Clin Endocrinol (Oxf) 2003; 58: 763-769

[19] Noh TW, Jeong $\mathrm{H}$, Lee MK et al. Predicting recurrence of nonfunctioning pituitary adenomas. J Clin Endocrinol Metab 2009; 94: 4406-4413

[20] Widhalm G, Wolfsberger S, Preusser M et al. Residual nonfunctioning pituitary adenomas: Prognostic value of MIB-1 labeling index for tumor progression. J Neurosurg 2009; 111: 563-571
[21] Edwards AA, Swords FM, Plowman PN. Focal radiation therapy for patients with persistent/recurrent pituitary adenoma, despite previous radiotherapy. Pituitary 2009; 12: 30-34

[22] Gittoes NJ. Radiotherapy for non-functioning pituitary tumors-when and under what circumstances? Pituitary 2003; 6: 103-108

[23] Fadul CE, Kominsky AL, Meyer LP et al. Long-term response of pituitary carcinoma to temozolomide. Report of two cases. J Neurosurg 2006; 105: 621-626

[24] Lim S, Shahinian H, Maya MM et al. Temozolomide: a novel treatment for pituitary carcinoma. Lancet Oncol 2006; 7: 518-520

[25] Syro LV, Uribe H, Penagos LC et al. Antitumour effects of temozolomide in a man with a large, invasive prolactin-producing pituitary neoplasm. Clin Endocrinol (Oxf) 2006; 65: 552-553

[26] Ji Y, Vogel RI, Lou E. Temozolomide treatment of pituitary carcinomas and atypical adenomas: Systematic review of case reports. NeuroOncol Pract 2016; 3: 188-195

[27] Losa M, Mazza E, Terreni MR et al. Salvage therapy with temozolomide in patients with aggressive or metastatic pituitary adenomas: Experience in six cases. Eur J Endocrinol 2010; 163: 843-851

[28] Bush ZM, Longtine JA, Cunningham T et al. Temozolomide treatment for aggressive pituitary tumors: correlation of clinical outcome with $\mathrm{O}(6)$-methylguanine methyltransferase (MGMT) promoter methylation and expression. J Clin Endocrinol Metab 2010; 95: E280-E290

[29] Raverot G, Sturm N, de Fraipont F et al. Temozolomide treatment in aggressive pituitary tumors and pituitary carcinomas: A French Multicenter Experience. J Clin Endocrinol Metab 2010; 95: 4592-4599

[30] Hirohata T, Asano K, Ogawa $\mathrm{Y}$ et al. DNA mismatch repair protein (MSH6) correlated with the responses of atypical pituitary adenomas and pituitary carcinomas to temozolomide: the national cooperative study by the Japan Society for Hypothalamic and Pituitary Tumors. J Clin Endocrinol Metab 2013; 98: 1130-1136

[31] Bengtsson D, Schroder HD, Andersen M et al. Long-term outcome and MGMT as a predictive marker in 24 patients with atypical pituitary adenomas and pituitary carcinomas given treatment with temozolomide. J Clin Endocrinol Metab 2015; 100: 1689-1698

[32] Ceccato F, Lombardi G, Manara R et al. Temozolomide and pasireotide treatment for aggressive pituitary adenoma: Expertise at a tertiary care center. J Neurooncol 2015; 122: 189-196

[33] Bruno OD, Juárez-Allen L, Christiansen SB et al. Temozolomide therapy for aggressive pituitary tumors: Results in a small series of patients from Argentina. Int J Endocrinol 2015; 1-8

[34] Losa M, Bogazzi F, Cannavo S et al. Temozolomide therapy in patients with aggressive pituitary adenomas or carcinomas. J Neuro-Oncol 2016; 126: 519-525

[35] Lasolle H, Cortet C, Castinetti F et al. Temozolomide treatment can improve overall survival in aggressive pituitary tumors and pituitary carcinomas. Eur J Endocrinol 2017; 176: 769-777

[36] Jordan JT, Miller J], Cushing T et al. Temozolomide therapy for aggressive functioning pituitary adenomas refractory to surgery and radiation: a case series. Neurooncol Pract 2018; 5: 64-68

[37] McCormack A, Dekkers OM, Petersenn S et al. Treatment of aggressive pituitary tumours and carcinomas: Results of a European Society of Endocrinology (ESE) survey 2016. Eur J Endocrinol 2018; 178: 265-276

[38] Heaney AP. Clinical review: Pituitary carcinoma: Difficult diagnosis and treatment. J Clin Endocrinol Metab 2011; 96: 3649-3660

[39] Kaltsas GA, Mukherjee J], Plowman PN et al. The role of cytotoxic chemotherapy in the management of aggressive and malignant pituitary tumors. J Clin Endocrinol Metab 1998; 83: 4233-4238

[40] Brabander T, Teunissen JJM, Van Eijck CH] et al. Peptide receptor radionuclide therapy of neuroendocrine tumours. Best Pract Res Clin Endocrinol Metab 2016; 30: 103-114

[41] Strosberg J, El-Haddad G, Wolin E et al. Phase 3 Trial of 177Lu-Dotatate for midgut neuroendocrine tumors. N Engl J Med 2017; 376: 125-135 
[42] Acosta-Gómez M], Muros MA, Llamas-Elvira JM et al. The role of somatostatin receptor scintigraphy in patients with pituitary adenoma or post-surgical recurrent tumours. $\mathrm{Br}$ J Radiol 2005; 78: 110-115

[43] Boni G, Ferdeghini M, Bellina CR et al. [111In-DTPA-D-Phe]-octreotide scintigraphy in functioning and non-functioning pituitary adenomas. Q J Nucl Med 1995; 39: 90-93

[44] Borson-Chazot F, Houzard C, Ajzenberg C et al. Somatostatin receptor imaging in somatotroph and non-functioning pituitary adenomas: Correlation with hormonal and visual responses to octreotide. Clin Endocrinol (Oxf) 1997; 47: 589-598

[45] Colao A, Lastoria S, Ferone D et al. The pituitary uptake of (111) In-DTPA-D-Phe1-octreotide in the normal pituitary and in pituitary adenomas. J Endocrinol Invest 1999; 22: 176-183

[46] Duet M, Ajzenberg C, Benelhadj S et al. Somatostatin receptor scintigraphy in pituitary adenomas: A somatostatin receptor density index can predict hormonal and tumoral efficacy of octreotide in vivo. J Nucl Med 1999; 40: 1252-1256

[47] Görges R, Cordes U, Engelbach M et al. [Prediction of pharmacological effect of octreotide in acromegaly by means of $111 \mathrm{ln}$-pentetreotide scintigraphy and calculation of a pituitary uptake index]. Nuklearmedizin 1997; 36: 117-124

[48] Legovini P, De Menis E, Billeci D et al. 111 Indium-pentetreotide pituitary scintigraphy and hormonal responses to octreotide in acromegalic patients. J Endocrinol Invest 1997; 20: 424-428

[49] Oppizzi G, Cozzi R, Dallabonzana D et al. Scintigraphic imaging of pituitary adenomas: An in vivo evaluation of somatostatin receptors. J Endocrinol Invest 1998; 21: 512-519

[50] Plöckinger U, Reichel M, Fett U et al. Preoperative octreotide treatment of growth hormone-secreting and clinically nonfunctioning pituitary macroadenomas: Effect on tumor volume and lack of correlation with immunohistochemistry and somatostatin receptor scintigraphy. J Clin Endocrinol Metab 1994; 79: 1416-1423

[51] Rieger A, Rainov NG, Elfrich C et al. Somatostatin receptor scintigraphy in patients with pituitary adenoma. Neurosurg Rev 1997; 20: 7-12

[52] Schmidt M, Scheidhauer K, Luyken C et al. Somatostatin receptor imaging in intracranial tumours. Eur J Nucl Med 1998; 25: 675-686

[53] Tofani A, Cucchi R, Pompili A et al. 111ln-octreotide scintigraphy in pituitary adenomas. Q J Nucl Med 1995; 39: 94-97

[54] Boy C, Heusner TA, Poeppel TD et al. 68Ga-DOTATOC PET/CT and somatostatin receptor (sst1-sst5) expression in normal human tissue: Correlation of sst2 mRNA and SUVmax. Eur J Nucl Med Mol Imaging 2011; 38: 1224-1236

[55] Parghane RV, Agrawal K, Mittal BR et al. 68Ga DOTATATE PET/CT in a rare coexistence of pituitary macroadenoma and multiple paragangliomas. Clin Nucl Med 2014; 39: 91-93

[56] d’Amico A, Stąpór-Fudzińska M, Tarnawski R. CyberKnife radiosurgery planning of a secreting pituitary adenoma performed with ${ }^{68} \mathrm{Ga}$ DOTATATE PET and MRI. Clin Nucl Med 2014; 39: 1043-1044

[57] Xiao ], Zhu Z, Zhong $D$ et al. Improvement in diagnosis of metastatic pituitary carcinoma by 68Ga DOTATATE PET/CT. Clin Nucl Med 2015; 40: e129-e131

[58] Garmes HM, Carvalheira JBC, Reis F et al. Pituitary carcinoma: A case report and discussion of potential value of combined use of Ga-68 DOTATATE and F-18 FDG PET/CT scan to better choose therapy. Surg Neurol Int 2017; 8: 162

[59] Basu S, Ranade R, Hazarika S. 68Ga DOTATATE PET/CT of synchronous meningioma and prolactinoma. Clin Nucl Med 2016; 41: 230-231

[60] Gauthé M, Sarfati J, Bourcigaux $N$ et al. Pituitary adenoma recurrence suspected on central hyperthyroidism despite empty sella and confirmed by 68Ga-DOTA-TOC PET/CT. Clin Nucl Med 2017; 42: 454-455
[61] Kim S, Dillon WP, Hope TA et al. Ectopic thyroid-stimulating hormone-secreting pituitary adenoma of the nasopharynx diagnosed by 68Ga-DOTA-TATE PET/CT. World Neurosurg 2019; 125: 400-404

[62] Zhao X, Xiao J, Xing B et al. Comparison of (68)Ga DOTATATE to $18 F-F D G$ uptake is useful in the differentiation of residual or recurrent pituitary adenoma from the remaining pituitary tissue after transsphenoidal adenomectomy. Clin Nucl Med 2014; 39: 605-608

[63] Wang H, Hou B, Lu L et al. PET/MRI in the diagnosis of hormone-producing pituitary microadenoma: A prospective pilot study. J Nucl Med 2018; 59: 523-528

[64] Baldari S, Ferraù F, Alafaci $C$ et al. First demonstration of the effectiveness of peptide receptor radionuclide therapy (PRRT) with 111 In-DTPA-octreotide in a giant PRL-secreting pituitary adenoma resistant to conventional treatment. Pituitary 2012; (15) S57-S60

[65] Kumar Gupta S, Singla S, Damle NA et al. Diagnosis of Men-I Syndrome on (68)Ga-DOTANOC PET-CT and role of peptide receptor radionuclide therapy with (177)Lu-DOTATATE. Int J Endocrinol Metab 2012; 10: 629-633

[66] Kovacs GL, Goth M, Rotondo F et al. ACTH-secreting Crooke cell carcinoma of the pituitary. Eur J Clin Invest 2013; 43: 20-26

[67] Komor J, Reubi JC, Christ ER. Peptide receptor radionuclide therapy in a patient with disabling non-functioning pituitary adenoma. Pituitary 2014; 17: 227-231

[68] Maclean J, Aldridge M, Bomanji ] et al. Peptide receptor radionuclide therapy for aggressive atypical pituitary adenoma/carcinoma: Variable clinical response in preliminary evaluation. Pituitary 2014; 17: 530-538

[69] Novruzov F, Aliyev JA, Jaunmuktane Z et al. The use of (68)Ga DOTATATE PET/CT for diagnostic assessment and monitoring of (177)Lu DOTATATE therapy in pituitary carcinoma. Clin Nucl Med 2015; 40: 47-49

[70] Waligorska-Stachura J, Gut P, Sawicka-Gutaj N et al. Growth hormonesecreting macroadenoma of the pituitary gland successfully treated with the radiolabeled somatostatin analog (90)Y-DOTATATE: Case report. J Neurosurg 2016; 125: 346-349

[71] Giuffrida G, Ferraù F, Laudicella $R$ et al. Peptide receptor radionuclide therapy for aggressive pituitary tumors: A monocentric experience. Endocr Connect 2019; 8: 528-535

[72] Ortiz LD, Syro LV, Scheithauer BW et al. Anti-VEGF therapy in pituitary carcinoma. Pituitary 2012; 15: 445-449

[73] Touma W, Hoostal S, Peterson RA et al. Successful treatment of pituitary carcinoma with concurrent radiation, temozolomide, and bevacizumab after resection. J Clin Neurosci 2017; 41: 75-77

[74] Rotman LE, Vaughan TB, Hackney JR et al. Long-term survival after transformation of an adrenocorticotropic hormone-secreting pituitary macroadenoma to a silent corticotroph pituitary carcinoma. World Neurosurg 2019; 122: 417-423

[75] Dutta P, Reddy KS, Rai A et al. Surgery, octreotide, temozolomide, bevacizumab, radiotherapy, and pegvisomant treatment of an AIP Mutation-Positive Child. J Clin Endocrinol Metab 2019; 104: 3539-3544

[76] Jouanneau E, Wierinckx A, Ducray F et al. New targeted therapies in pituitary carcinoma resistant to temozolomide. Pituitary 2012; 15: 37-43

[77] Donovan LE, Arnal AV, Wang SH et al. Widely metastatic atypical pituitary adenoma with mTOR pathway STK11(F298L) mutation treated with everolimus therapy. CNS Oncol 2016; 5: 203-209

[78] Zhang D, Way JS, Zhang X et al. Effect of everolimus in treatment of aggressive prolactin-secreting pituitary adenomas. J Clin Endocrinol Metab 2019; 104: 1929-1936

[79] Cooper O, Mamelak A, Bannykh S et al. Prolactinoma ErbB receptor expression and targeted therapy for aggressive tumors. Endocrine 2014; 46: 318-327

[80] Lin AL, Jonsson P, Tabar $V$ et al. Marked response of a hypermutated acth-secreting pituitary carcinoma to ipilimumab and nivolumab. J Clin Endocrinol Metab 2018; 103: 3925-3930 